\title{
A Dosimetric Comparison Between 3D-Conformal and Intensity-Modulated Co-Planer Radiotherapy Plans for Treatment of Pediatric Posterior Fossa Medulloblastoma.
}

\author{
Wael S. Makar ${ }^{1}$ and Rania M. Mossa ${ }^{2}$. \\ ${ }^{1}$ Lecturer of Clinical Oncology, ${ }^{2}$ Department of Physics, Kasr El-Aini Center of Clinical Oncology \\ and Nuclear Medicine (NEMROCK).
}

\begin{abstract}
Background: Craniospinal irradiation followed by a boost to the posterior fossa combined with cisplatinbased chemotherapy after maximal safe resection is the standard of care in treatment of pediatric medulloblastoma. Sensorineural hearing loss is one of the most serious positble late effect of this treatment. Material and Method: Five high-risk medulloblastoma pediatric patients were treated by craniospinal irradiation (36 Gy) combined with cisplatin weekly followed by a boost of irradiation (18 Gy) to the posterior fossa by 3D-CRT plan. Dosimetric plans by IMRT were done to the 5 cases to compare between 3D-CRT and IMRT in sparing of the different OARs as well as their coverage of the target.

Results: By comparing the mean doses and DVHs for the 3D-CRT and IMRT plans, it was obvious that IMRT plans were able to spare the cochlea more than the 3D-CRT. As the cochlea received $80.5 \%$ (43.5 Gy) of the prescribed dose in the IMRT plans compared to $94.5 \%$ (51. Gy) of the prescribed dose in the 3D-CRT plans, even the target was better covered by IMRT as it has been shown when comparing the DVHs of the different plans.

Conclusion: IMRT increases the conformality of dose of radiation in the target and limiting the dose to the OARs especially the cochlea thus reducing the incidence of sensory hearing loss.
\end{abstract}

Key words: 3D-CRT, IMRT, medulloblastoma, cochlea. Corresponding Author: Wael Samir Makar

E-mail: wael_makar@yahoo.com

\section{INTRODUCTION}

Medulloblastoma accounts for about of $20 \%$ of primary CNS tumors in childhood. Current treatment strategies which include maximal safe resection, croniospinal irradiation followed by a boost to the posterior fossa with cisplatin based chemotherapy have resulted in approximately $80 \%$ and $70 \%$ 5 -year survival rates in standard-risk and high-risk medulloblastoma respectively ${ }^{1,2}$.

As survival rates in children with medulloblastoma improve, the issue of late effects of treatment becomes more important. One possible consequence of treatment with cisplatin-based chemotherapy and radiotherapy is sensorineural hearing loss, this clinically important side effect, which can cause difficulties in speech, language, communication and learning must be considered during treatment planning. Characteristically, sensorineural hearing loss occurs 6-12 months following radiotherapy and can be irreversible ${ }^{3}$.

Patterns of treatment failure for medulloblastoma have been explored by several investigators in different studies showed that the most common site of relapse is the posterior fossa itself, according to that, local disease control in posterior fossa is directly related to radiation dose, in contrast to craniospinal axis radiotherapy ${ }^{4}$.

Three-dimensional conformal radiotherapy (3D-CRT) has been shown to be superior to twodimensional radiotherapy in conforming the high dose volume closely to planning target volume without increasing doses to the inner ear structures ${ }^{5}$.

Intensity-modulated radiation therapy (IMRT) is the product of advances in the technology of radiotherapy to deliver radiation more precisely to the tumor while relatively limiting dose to the surrounding normal tissues. IMRT can increase the conformality of dose distributions, particularly for concave targets and may improve tumor control while reducing normal tissue toxicity ${ }^{6}$.

Beam exit doses from multiple portals increase the integral dose to adjacent normal tissues and may lead to growth disturbances in children and increase the risk of secondary malignancies. IMRT often produces greater whole body doses because the longer irradiation times produce increased head scatter from the linear accelerator 6 . 
This study compares dosimetrically 3D-CRT and IMRT planning in posterior fossa treatment in pediatric medulloblastoma as regard the target coverage, their potential to reduce ototoxicity by limiting the dose to the cochlea and other organs at risk (OARs).

\section{PATIENTS AND METHODS}

This study included 5 high risk posterior fossa medulloblastoma patients. Age range from 3 to 6 years old. All the included patients underwent surgical excision of the posterior fossa mass with application of a V-P shunt with a postoperative residual mass more than $1.5 \mathrm{~cm}$ in the maximum cross section diameter.

Patients were scanned and treated in the prone position in a thermoplastic shell which extended to the base of the skull to ensure reproducible postioning.

The patients received cranio-spinal irradiation as phase I of radiotherapy in a dose of 36 Gy given in 20 fractions over 4 weeks with cisplatin weekly as a radiosensitizer.

Phase II of radiotherapy included the whole posterior fossa with a presecribed dose of 18 Gy given in 10 fraction with a daily fraction of $1.8 \mathrm{~Gy}$, also with weekly cisplatin so that the posterior fossa received a total dose of $54 \mathrm{~Gy}$.

The whole posterior fossa was regarded as the Clinical Target Volume (CTV). The posterior fossa was defined by the posterior clinoid anteriorly, the internal occipital protuberance posteriorly, the superior border was defined by the cerebeller tentorium and inferior border by the junction of the first and second cervical vertebrae. The Planning Target Volume (PTV) was constructed by adding a three dimensional margin of $5 \mathrm{~mm}$ around the CTV. Organs at risk (OARs) included the non-posterior fossa, pituitary, cochlea, eyes and optic nerves.

Table 1: Shows the mean, maximum and minimum doses in Gy to the PTV and OARs in 3D CRT plan.

\begin{tabular}{lccc}
\hline Organ & $\begin{array}{c}\text { Min. dose } \\
(\mathbf{G y})\end{array}$ & $\begin{array}{c}\text { Max. dose } \\
(\mathbf{G y})\end{array}$ & $\begin{array}{c}\text { Mean dose } \\
(\mathbf{G y})\end{array}$ \\
\hline PTV & 13.7 & 19.2 & 18.0 \\
\hline Rt. cochlea & 13.5 & 16.7 & 15.1 \\
\hline Lt. cochlea & 11.5 & 16.0 & 13.9 \\
\hline Rt. eye & 0.2 & 0.5 & 0.3 \\
\hline Lt. eye & 0.08 & 1.1 & 0.4 \\
\hline Rt. lens & 0.2 & 0.2 & 0.2 \\
\hline Lt. lens & 0.2 & 0.3 & 0.3 \\
\hline Optic nerve & 0.4 & 2.0 & 0.9 \\
\hline Chiasma & 5.0 & 16.9 & 13.2 \\
\hline Pituitary & 13.8 & 16.9 & 15.9 \\
\hline
\end{tabular}

Patients were actually treated by 3D-conformal plan consisting of a pair of wedged posterior oblique fields and the IMRT plan was constructed dosimetrically. The IMRT plan consisted of a five field intensity-modulated plan optimized with an inverse planning tool. The angles of the beams were arranged to cover the posterior fossa while minimizing dose to the cochlea as much as possible (Figures 1 and 2).

Plans were evaluated by comparison of dose distributions for the PTV and OARs. Dose-volume histograms (DVHs) were created for all outlined structures in each plan and dose staticatics were compared to the PTV and OARs.

\section{RESULTS}

The 3D-CRT plans were more on less identical in the 5 cases treated in our study as regard the beam arrangements, dose distribution to the PTV and OARs.

As it is obvious from the previous tables, the cochlea received $80.5 \%$ (43.5 Gy) of the prescribed dose in the IMRT plans compared to $94.5 \%$ (51.0 Gy) of the prescribed dose in the 3D-CRT plans and this predicts a significant lower rate of grade 3-4 ototoxicity by using the IMRT technique. Knowing that sensorinenal hearing loss can occur at doses above 50-60 Gy to the cochlea.

Also, the pituitary gland dose was significantly lower in the IMRT plans 46 Gy compared to 52 Gy in the 3D-CRT plans.

Dose volume histograms (DVHs) of the 3D-CRT plan and IMRT plans are shown in Figure (3, 4 and 5).

Table 2: Shows the mean, maximum and minimum doses in Gy to the PTV and OARs in the IMRT plan.

\begin{tabular}{lccc}
\hline Organ & $\begin{array}{c}\text { Min. dose } \\
(\text { Gy) }\end{array}$ & $\begin{array}{c}\text { Max. dose } \\
(\text { Gy })\end{array}$ & $\begin{array}{c}\text { Mean dose } \\
(\text { Gy) }\end{array}$ \\
\hline PTV & 11.9 & 19.8 & 17.9 \\
\hline Rt. cochlea & 6.0 & 9.5 & 7.5 \\
\hline Lt. cochlea & 5.7 & 10.6 & 7.5 \\
\hline Rt. eye & 0.1 & 2.9 & 0.8 \\
\hline Lt. eye & 0.04 & 2.1 & 0.5 \\
\hline Rt. lens & 0.2 & 0.3 & 0.2 \\
\hline Lt. lens & 0.2 & 0.3 & 0.2 \\
\hline Optic nerve & 1.1 & 3.4 & 2.5 \\
\hline Chiasma & 3.6 & 15.6 & 8.3 \\
\hline Pituitary & 7.2 & 13.8 & 9.9 \\
\hline
\end{tabular}


Kasr-El-Aini Journal Of Clinical Oncology And Nuclear Medicine

Vol. 7 | No. 3-4 2011

A Dosimetric Comparison Between 3D-Conformal and...

Table 3: Shows the difference between the mean doses in Gy in the 3D-CRT and IMRT plans*.

\begin{tabular}{|c|c|c|}
\hline Organ $\quad$ Plan & $\begin{array}{c}\text { 3D-CRT } \\
\text { Mean dose (Gy) }\end{array}$ & $\begin{array}{c}\text { IMRT } \\
\text { Mean dose (Gy) }\end{array}$ \\
\hline PTV & 18.0 & 17.9 \\
\hline Rt. Cochlea & 15.1 & 7.5 \\
\hline Lt. cochlea & 13.9 & 7.5 \\
\hline Rt. Eye & 0.3 & 0.8 \\
\hline Lt. eye & 0.4 & 0.5 \\
\hline Rt. Lens & 0.2 & 0.2 \\
\hline Lt. lens & 0.3 & 0.2 \\
\hline Optic nerve & 0.9 & 2.5 \\
\hline Chiasma & 13.2 & 8.3 \\
\hline Pituitary & 15.9 & 9.9 \\
\hline
\end{tabular}

*Standard deviation (SD) for all variables was zero so no statistics could be done

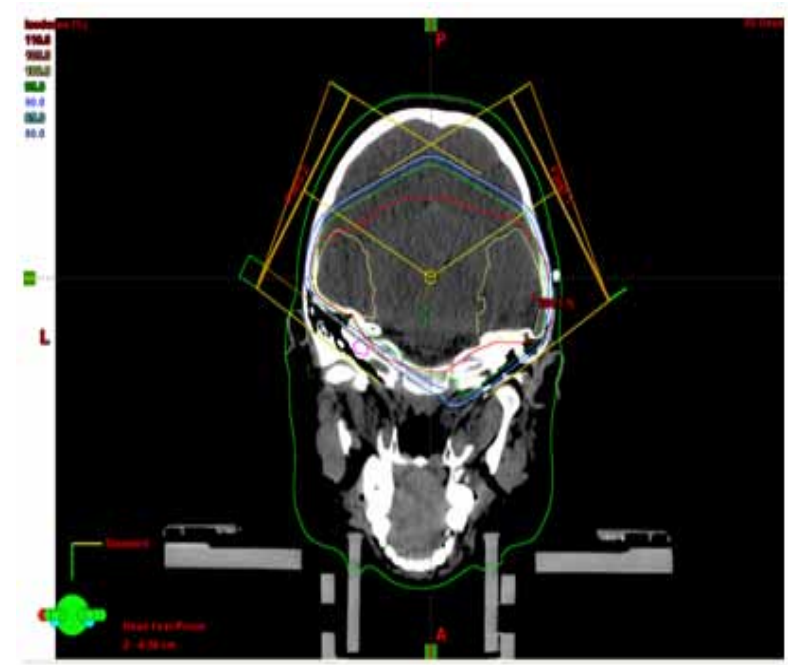

Figure 1: Beam arrangement in 3D-CRT plan.

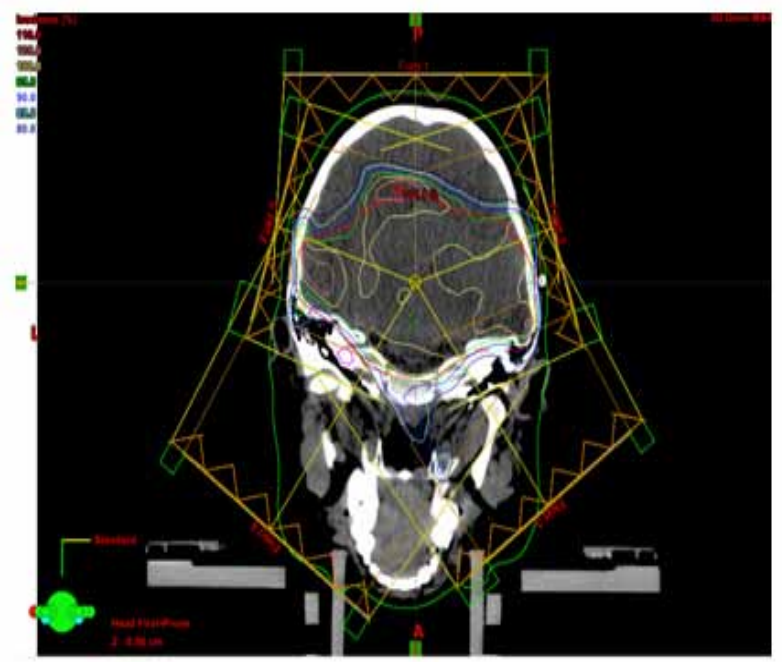

Figure 2: Beam arrangement in IMRT plan.

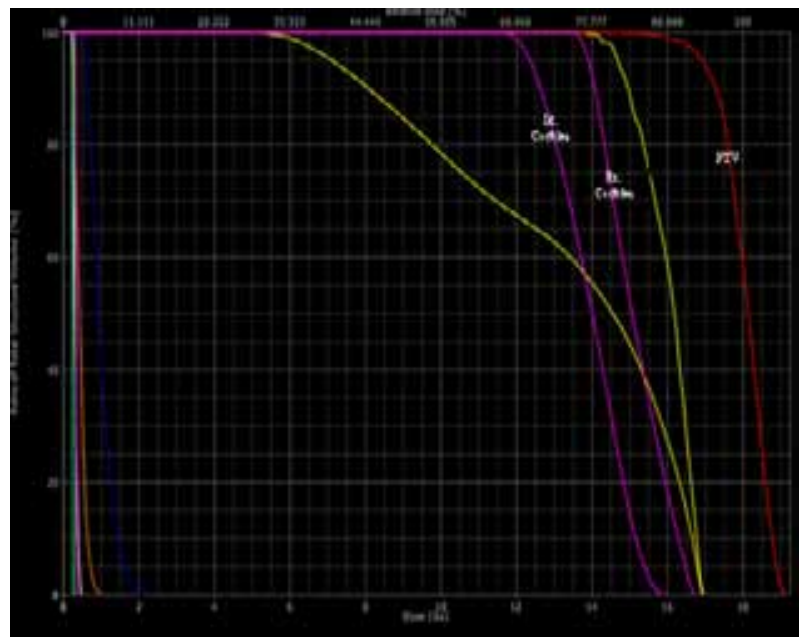

Figure 3: DVH of the 3D-CRT plan.

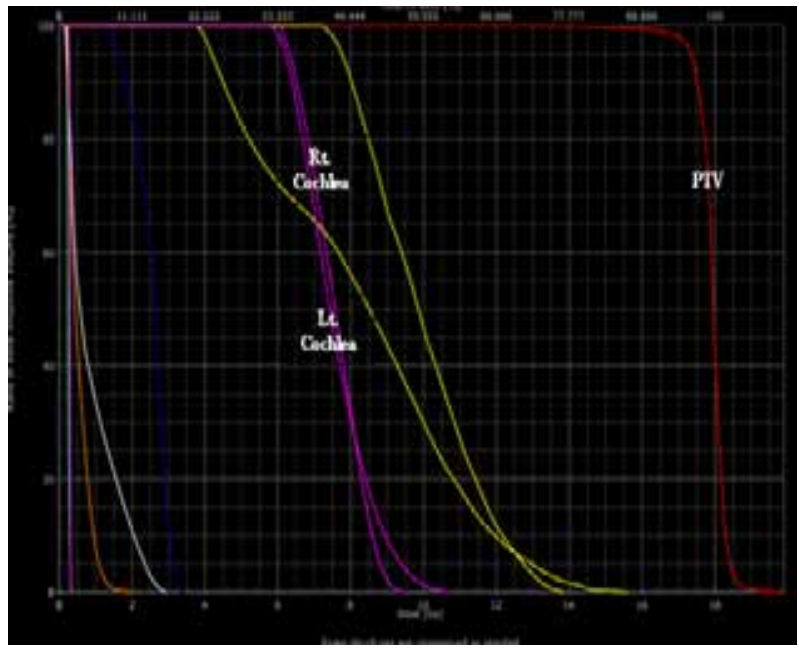

Figure 4: DVH of the IMRT plan.

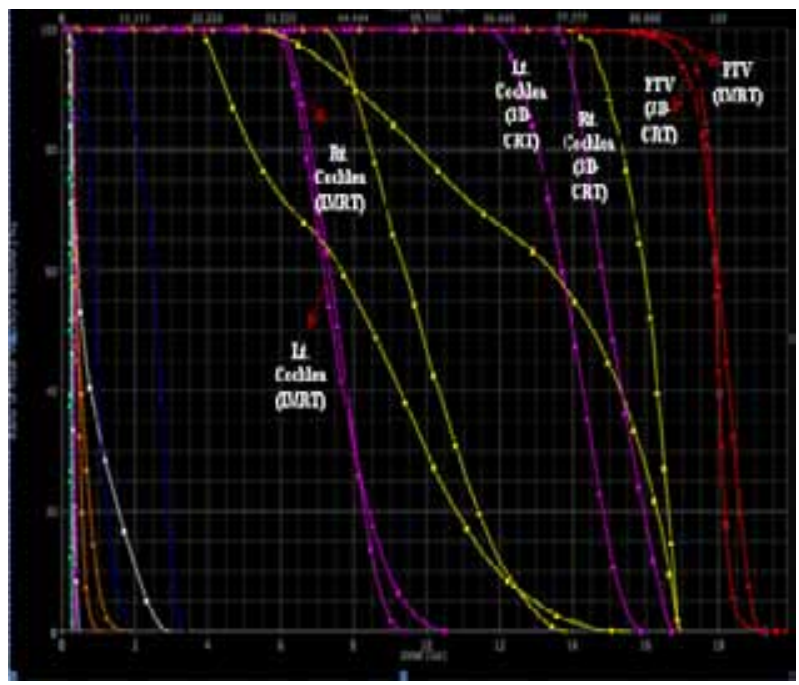

Figure 5: DVH of both the 3D-CRT and IMRT plans. 


\section{DISCUSSION}

This dosimetric planning study has compared the two 3D radiotherapy techniques namely the 3D-CRT and IMRT, for the treatment of the posterior fossa in five pediatric patients. Both techniques were able to provide acceptable coverage of the target volume. However, sparing of the dominant OAR mainly the cochlea was achieved more with the IMRT technique.

Huang et $a l^{3}$ compared conventional parallelopposed fields with IMRT plans. The IMRT plans demonstrated cochlear sparing, delivering less than $70 \%$ of the prescribed dose to the cochlea. IMRT not only reduced the dose to the auditory apparatus below its tolerance limit but it simultaneously delivered a higher dose to the target.

Fukunaga-Johnson et $a l^{4}$ composed the dose distributions in the target volume (posterior fossa) and the cochlea for the conventional 2D technique (parallelapposed lateral fields) and a 3D-CRT cochlear sparing technique (a wedged pair of posterior oblique fields). They found that, while the standard 2D technique delivered the prescribed dose to both cochlea, the 3D-CRT plan reduced the cochlear dose to $<70 \%$ of the prescribed dose.

Almost the same results demonsterated by Hua et al. ${ }^{7}$ in a study that compared the conventional, conformal and IMRT for posterior fossa treatment and found that 3D-CRT and IMRT plans reduced cochlear doses to less than $70 \%$ of the mean target dose while it maintained adequate target coverage.

Plowman et al. $^{8}$ found the increased cochlearsparing effect provided by IMRT were minimal when compared with the 3D-CRT. When limiting the field arrangement to coplanar beams, a minimum of four beams were required to meet treatment planning goals. While the use of as many as six posterior beams did improve target dose homogeneity, exit doses in the anterior half of the head were increased. The fieldmodulating properties of IMRT were not able to provide sufficient cochlear sparing when the beams entered the head through the cochlea, thus, beam entry was restricted to the posterior portion of the head.

Paulino et al. ${ }^{7}$ conducted a study to determine whether the use of cochlear-sparing IMRT boost results in excess local failures in children with medulloblestoma and found that the use of IMRT was associated with excellent local control and did not result in excess posterior fossa failures outside of the tumor bed.

Our results reinforce the findings of these investigators, that IMRT plans can reduce the dose to the cochlea more than the 3D-CRT and definitely more than the $2 \mathrm{D}$ conventional techniques. But, the increase in monitor units (MD) associated with IMRT results in greater head scatter in the linear accelerator and therefore greater whole body dose. This can have implications for the pediatric patients. Furthermore, IMRT is a very resource-consuming radiotherapy technique, as prescription times are increased due to the need for contouring OARs, treatment planning times are increased due to the time required for optimizing the beam fluences and at present, the time needed for treatment validation because of the complex relationship between linac output and dose at a point in a modulated field. The implementation of such a resourceintensive technique may be beyond the means of many centers.

In conclusion, IMRT is an effective technique in increasing the conformality of the dose of radiation in the target and limiting the dose to the OARs especially the cochlea thus reducing the incidence of sensory hearing loss. But, it is rather a complex technique that needs further researches with greater number of cases and longer follow-up to confirm this conclusion and detect the risk of developing secondary malignancies.

\section{REFERENCES}

1. Packer RJ, Goldwein J, Nicholson HS, Vezina LG, Allen JC, Ris MD, et al. Treatment of children with medulloblastomas with reduced-dose craniospinal radiation therapy and adjuvant chemotherapy: A Children's Cancer Group study. J.Clin.Oncol. 1999;17(7):2127-36.

2. McNeil DE, Coté TR, Clegg L, Mauer A. SEER update of incidence and trends in pediatric malignancies: Acute lymphoblastic leukemia. Med.Pediatr.Oncol. 2002;39(6):554-7.

3. Huang E, Teh BS, Strother DR, Davis QG, Chiu JK, $\mathrm{Lu} \mathrm{HH}$, et al. Intensity-modulated radiation therapy for pediatric medulloblastoma: Early report on the reduction of ototoxicity. Int.J.Radiat.Oncol.Biol.Phys. 2002;52(3): 599-605.

4. Fukunaga Johnson N, Lee JH, Sandler HM, Robertson P, McNeil E, Goldwein JW. Patterns of failure following treatment for medulloblastoma: Is it necessary to treat the entire posterior fossa? Int.J.Radiat.Oncol.Biol.Phys. 1998;42(1):143-6.

5. Fukunaga Johnson N, Sandler HM, Marsh R, Martel MK. The use of 3D conformal radiotherapy (3D CRT) to spare 
the cochlea in patients with medulloblastoma. Int.J.Radiat. Oncol.Biol.Phys. 1998;41(1):77-82.

6. Purdy JA. Intensity-modulated radiotherapy: Current status and issues of interest. Int.J.Radiat.Oncol.Biol.Phys. 2001;51(4):880-914.

7. Hua C, Bass JK, Khan R, Kun LE, Merchant TE. Hearing loss after radiotherapy for pediatric brain tumors: Effect of cochlear dose. Int.J.Radiat.Oncol.Biol.Phys. 2008;72(3):892-9.
8. Breen SL, Kehagioglou P, Usher C, Plowman PN. A comparison of conventional, conformal and intensitymodulated coplanar radiotherapy plans for posterior fossa treatment. Br.J.Radiol. 2004;77(921):768-74.

9. Paulino AC, Mazloom A, Teh BS, South M, Okcu MF, $\mathrm{Su} \mathrm{J}$, et al. Local control after craniospinal irradiation, intensity-modulated radiotherapy boost and chemotherapy in childhood medulloblastoma. Cancer 2011;117(3): 635-41. 